Chapter 4

\title{
Porcine Model of Huntington's Disease
}

\author{
Petra Rausova, Petra Vochozkova, Daniela Vidinska, \\ Eva Hrnciarova, Bozena Bohuslavova, \\ Monika Macakova, Ivona Valekova, Stefan Juhas, \\ Taras Ardan, Petr Solc, Jan Motlik and \\ Zdenka Ellederova
}

Additional information is available at the end of the chapter

http://dx.doi.org/10.5772/66353

\begin{abstract}
At present, we are probably the only research facility to be breeding transgenic Huntington's disease minipigs (TgHD). These minipigs express N-terminal part of human mutated huntingtin including $124 \mathrm{Q}$ under the control of human huntingtin promoter. The founder animal, born in 2009, gave birth to four subsequent generations with an equal contribution of wild-type (WT) and transgenic (TgHD) piglets in all litters. We take different approaches, some of which are unique for large animal models, to study the phenotype development comparing WT and TgHD siblings. In this chapter, we review these approaches and the phenotype progression in the minipigs. Additionally, we outline perspectives in generation of new models using novel methodology and the potential of pig models in preclinical HD studies.
\end{abstract}

Keywords: large animal models, pig, huntingtin, transgenic animal, Huntington's disease

\section{Introduction}

The cause of Huntington's disease (HD), an abnormal polyglutamine elongation of the gene encoding the huntingtin protein (Htt), is known for more than two decades, but the effective treatment is still lacking. Animal models of Huntington's disease represent an indispensable part of disease investigation. It offers better understanding of molecular mechanisms involved 
in the disease pathology and above all testing novel potential therapeutic approaches in HD treatment. Many different HD animal models were created. Predominantly used rodent models represent an important tool for understanding the complex progression of HD phenotype and have been used for many preclinical studies. However, the rodent's small brain size, differences in neuroanatomy to humans and short lifespan limit their application for detailed modelling of the pathogenic features of human disease. Moreover, wider scale of models is desired especially for safety and tolerability tests of potential therapeutics and longitudinal studies of HD.

In consequence, large animal models of HD, including non-human primate [1], sheep [2] and pigs [3-5], have been generated. The advantages of pigs, in particular the minipigs, are the adult body weight of 70-90 kg, relatively large gyrencephalic brain with similar neuroanatomy to humans, longer lifespan of 12-15 years and other anatomical, physiological and metabolic similarities to humans [6]. Because of their sophisticated cognitive and motor abilities, pigs are suitable for longitudinal learning, memory and behavioural studies. Furthermore, pigs are excellent models for disease progression studies, because of their long lifespan. The pig brain size and neuroanatomy make them available to be used in neurosurgical procedures and noninvasive imaging methods similar to those used in human diagnostics $[7,8]$. In addition, pigs are farm animals, and thus, the social tolerance for using them as experimental animals is higher than in other large animal models such as non-human primates or animals regularly kept as pets. Moreover, the minipig's litter size is usually six to eight pups, thus providing a good experimental group. Hence, the creation of HD minipig model offers many advantages for the HD studies.

Up to now, three attempts to generate HD transgenic pig models have been reported. In 2001, porcine mutant huntingtin (75Q) cDNA was microinjected in the pronucleus of fertilized eggs [3]. Several copies of the transgene were incorporated in the porcine genome, but there might have been problems with the gene intactness or transcriptional silencing. No HD phenotype was reported. In 2010, HD transgenic minipig expressing the N-terminal part of human mutated huntingtin with $105 \mathrm{Q}$ was created using the somatic cell nuclear transfer strategy (N208-105Q) [4], but these piglets died $53 \mathrm{~h}$ after birth, which could be due to the incomplete reprogramming during somatic cells nuclear transfer.

The transgenic HD minipig (TgHD) model created in Libechov expresses N-terminal part of human mutated huntingtin (N548-124Q) under the control of human huntingtin promoter. It was generated using strategy based on lentiviral infection of porcine embryos [5]. The mutant huntingtin was detected at the level of RNA and protein in central nervous system (CNS) as well as in peripheral tissues. The fluorescent in situ hybridization (FISH) and genomic analysis confirmed the incorporation of one copy of mutant huntingtin (mHTT) into non-coding sequence of the first porcine chromosome (1q24-q25) not interrupting any coding sequence in the pig genome. However, TgHD minipigs possess both endogenous alleles coding wild-type $\mathrm{Htt}$ (wtHtt). Because the reducing amount of wtHtt has substantial influence on disease development in HD patients [9], the physiological level of wtHtt in TgHD minipigs may postpone a phenotype progression. Most of the minipigs are now in the preclinical stage of HD. However, we observe the disease progression in the oldest animals based on behavioural, immunological, immunohistological and biochemical methods. 
Our transgenic minipig model represents an advantageous model for studying wide range of aspects of HD like molecular mechanisms of HD in primary cells isolated from TgHD and WT animals, brain and other organ structure using high-resolution imaging techniques or postmortem, preclinical symptoms of the disease and longitudinal non-invasive studies. Importantly, the minipig model is feasible for preclinical therapeutic studies (unpublished results) and thus can serve as the link between rodents and humans.

\section{Impairment of male fertility: testes and sperm pathology}

Although HD is characterized mainly by neurodegeneration, we at first discovered a reproductive failure in TgHD boars, starting at the age of 13 months and worsening with age.

In general, wild-type and mutant forms of $\mathrm{Htt}$ are expressed in many tissues, mostly in brain and testes [10]. Furthermore, the testes and brain display the most comparable gene expression pattern compared to other tissues [5]. Testicular degeneration was a long time unknown feature of HD. Interestingly, the first reports of testicular degeneration were described in mouse models. R6/2 mice showed dramatic atrophy of testes, which started at 4 weeks of age [11]. Also in YAC128 mouse models, mHtt causes testicular atrophy and male fertility problems occurring before neurodegeneration [12-14]. In human patients, only analysis of testes from post-mortem samples was performed [13]. This study showed a decreased number of spermatocytes and spermatids in HD patients; the seminal tubules of the patients were thicker than the seminal tubules of the healthy controls.

In our TgHD minipig model, we observed reduction in spermatozoa [5] and also their functional defect. In vitro penetration assay showed impairment of TgHD spermatozoa to penetrate oocytes with intact zona pellucida, which was in accordance with lower sperm motility and progressivity. Electron microscopy (EM) revealed sperm and testicular morphology defects. Deformity of nucleus associated with incomplete chromatin condensation, abnormal acrosome, and also the absence of residual bodies were seen in TgHD spermatozoa. Proximal cytoplasmic droplets were often associated with disorganized mitochondrial sheaths (Figure 1). The presence of the polyglutamine-containing proteins was observed in structures in the spermatozoa tail of TgHD boars using immunocytochemistry (ICC) and Western blot (WB) [14].

Moreover, detailed examination of TgHD testes showed degenerative changes in seminiferous tubules. Apoptotic spermatogonia and Sertoli cells were detected. EM of 24- and 36-monthold boars showed degenerative changes. The increased density of cytoplasm of Sertoli cells associated with its vacuolization, swollen mitochondria and dilated endoplasmic reticulum and clumps of heterochromatin in the nucleus were observed. Lamina basalis was often thick and undulated, made up of several layers due to the reduction in tubules diameter in the absence of spermatogenic elements (Figure 2). Seminiferous tubules of testes from the WT siblings were intact [14]. 


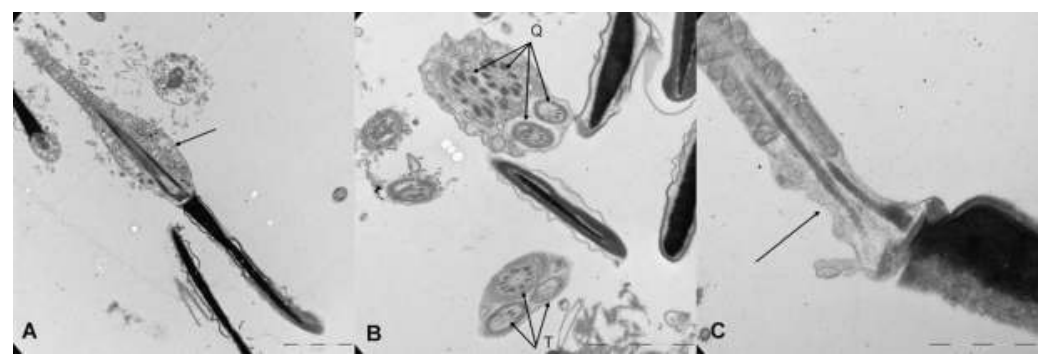

Figure 1. Morphologic defects of spermatozoa in TgHD boar's sperm. (A) Cytoplasmic droplet on midpiece of sperm. (B) Double, triple-T, quadruple-Q axoneme with fused mitochondrial sheaths. (C) Break on midpiece of sperm tail.

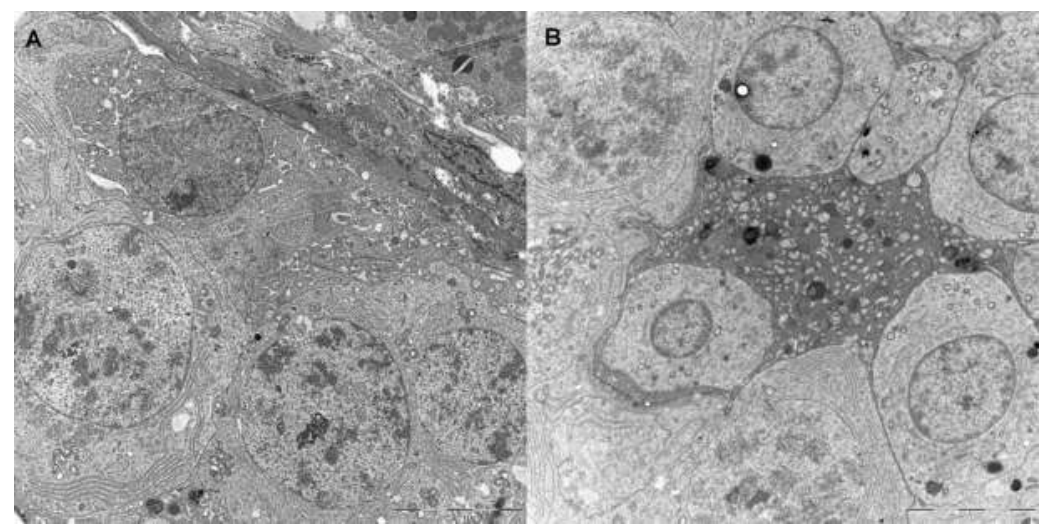

Figure 2. Seminiferous epithelium of TgHD boar at the age of 36 months. (A) Spermatogenic cells and (B) Sertoli cell undergoing apoptosis, increased chromatin condensation, increased density and vacuolization of cytoplasm.

In addition, the testes from 24-month-old TgHD boars were analysed using non-invasive methodology of 31P magnetic resonance spectroscopy (MRS) [15]. The results of this study showed significant reduction in relative phosphodiester (PDE) concentration in testicular parenchyma of TgHD boars compared to wild-type ones of the same ages. A decreased level of PDE $/ \gamma$-ATP ratio in TgHD minipigs may be related to decreased concentration of seminal fluid or the changes in sperm motility. This hypothesis agrees with the observed sperm pathology in both our previous studies discussed above [5, 14].

\section{Markers of neurodegeneration}

\subsection{Aggregates and fragmentation}

It is known that the most affected organ in HD is the brain, especially the medium-sized spiny neurons in striatum, and the pyramidal cells in the cortex are disrupted [16]. The hallmark of 
the disease is an accumulation of misfolded proteins, resulting in the formation of aggregates [17]. Nevertheless, the precise role of the aggregates in pathogenesis of HD is still not clear. Recent studies suggest their protective role against the effects of $\mathrm{mHtt}$ [18]. Moreover, smaller soluble forms of $\mathrm{mHtt}$ and huntingtin oligomers were described to be toxic to the cells and to be the key factors of cellular dysfunction [19]. Furthermore, the inhibition of the mHtt proteolysis reduces neurotoxicity [20]. In affected areas of the brain in human patients, the expanded $\mathrm{Htt}$ is found rather in fragmented, oligomerized and polymerized forms [21].

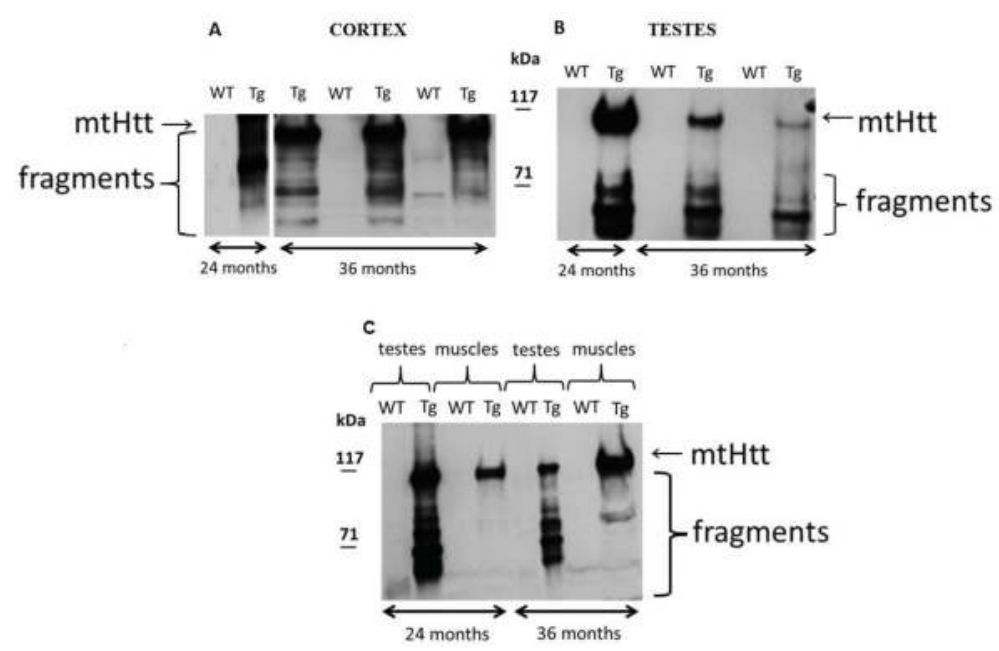

Figure 3. Fragmented mHtt detected in 24- and 36-month-old TgHD minipigs compared to their WT siblings in different tissues by polyQ 3B5H10 antibody. Western blot analysis shows fragmented forms of mHtt in (A) cortex and (B) testes of TgHD minipigs. (C) Comparing to muscles of TgHD minipigs, testes show increased amount of fragmented $\mathrm{mHtt}$ of 24- as well as 36-month-old minipigs. Reprinted with permission of CzMA JEP adopted from CeskSlovNeurol N2015; 78/111 (Suppl 2).

In our TgHD minipig model, we continuously test the aging animals for aggregates by several methods, which have been previously used in other animal models. The main focus is on the visualization of aggregates by immunohistochemistry (IHC) using anti-Htt antibodies, some of which are specific to higher molecular formation. For example, MW8 is a commercial antibody against N-terminal end, recognizing alpha-helical, random coil and extended conformations of huntingtin. In addition to the antibodies, certain dyes like Congo red stain protein aggregate by binding to fibrils with enriched $\beta$-sheet conformation $[22,23]$. Another biochemical method suitable for detection of aggregates is the filter retardation assay based on the fact that very large polymers cannot pass through a $0.2-\mu \mathrm{m}$ cellulose acetate filter and therefore can be identified [24]. Seprion assay uses beads binding amyloid structures, which can be then revealed on WB [25]. Velocity sedimentation method is based on fractionation of the sample by ultracentrifuge and detection of proteins in different fractions by SDS-PAGE and WB [26]. Htt oligomers and monomers can be detected by WB, taking advantage of oligomer retardation in stacking gel. Also, SDS agarose gel electrophoresis (AGERA) [27], 1\% 
agarose gel with a lower SDS concentration $(0.1 \%)$ without reducing agent and heating of the samples, can be used. Another approach is an immunoprecipitation with oligomer-specific antibodies (OC, A11). In order to distinguish between soluble and insoluble forms stabilized by covalent bonds, formic acid that cannot dissolve covalent but can dissolve non-covalent bonds can be used.

Up to date, we have detected only a very few aggregates in 24- and 36-month-old TgHD brain sections by IHC (unpublished data) in comparison with the massive incidence of aggregates in the R6/2 mice brain sections. We suspect that the aggregate formation will progress in older animals. Nevertheless, we could detect $\mathrm{N}$-terminal $\mathrm{mHtt}$ fragments in 24 months of age in brain and testes increasing with the age. Interestingly, most of the other tissues such as heart and muscles do not show fragmentation at 24 months. Only a small amount of fragments can be detected in muscles of 36-month-old animals, suggesting progression of the disease with age (Figure 3) [28]. Furthermore, we detected smears in the stacking gels of 3-8\% polyacrylamide gels in TgHD cortex and testes samples, also starting at 24 months of age [28].

\subsection{Magnetic resonance imaging (MRI) and spectroscopy (MRS)}

Neurodegeneration in minipigs can be observed by several approaches. One of them is a noninvasive approach by MRI. MRI including objective motor measures (Q-motor) showed a relationship between a decrease in brain volume and progression of HD in patients [29-31]. It was shown that MRI applicable for the brain volume assessment can be performed also with Libechov TgHD and WT minipigs [32].

Magnetic resonance spectroscopy (MRS), also known as nuclear magnetic resonance (NMR) spectroscopy, is a non-invasive method used in research and clinical practice that allows an evaluation of in vivo metabolism at the molecular level [33]. Concentrations of metabolites, such as $\mathrm{N}$-acetylaspartate, creatine, phosphocreatine, glutamate, glutamine, choline-containing compounds, inositol, $\gamma$-aminobutyric acid and others, can be determined by ${ }^{1} \mathrm{H}$ MRS [34]. ${ }^{1} \mathrm{H}$ MRS has previously been considered as a biomarker method in pre-manifest and early stages of HD [35-37]. In order to evaluate in vivo brain metabolite differences, single-voxel spectroscopy (SVS) has been primarily used [35, 38, 39]. However, several studies have also used two-dimensional chemical shift imaging (2D-CSI) [33, 40,41]. Studies measuring changes by MRS revealed different values of metabolite concentrations in patients with $\operatorname{HD}[34,35,42$, 43]. Recently, several longitudinal clinical studies have been performed. They showed a decrease in creatine and other metabolites (myo-inositol, $\mathrm{N}$-acetylaspartate and choline) in striatum, white matter axial diffusivity and connectome changes in HD gene carriers during disease onset [44-46]. Various changes in brain metabolite concentration have also been found in different HD animal models (mice, non-human primates). Moreover, our scientific team also determined changes in the brain of TgHD minipigs before HD onset using ${ }^{1} \mathrm{H}$ magnetic resonance (MR) spectroscopy. Measurements were performed on a $3 \mathrm{~T}$ MR scanner using a single-voxel spectroscopy sequence for spectra acquisition in the white matter and chemical shift imaging sequence for measurement in the striatum, hippocampus and thalamus. Similarly to HD patients and HD animal models, we revealed significant decrease in total creatine $(\mathrm{tCr})$ in the thalamus of 2-year-old TgHD boars accompanied with a non-significant 
decrease in $\mathrm{tCr}$ in all examined brain areas. This aspect resulted into significant changes in metabolite ratios (increased metabolic ratios of total choline $\mathrm{tCho} / \mathrm{tCr}$ in the striatum, thalamus, hippocampus as well as white matter). Creatine represents an important marker for brain energy metabolism, and we had supposed that the majority of the observed changes were predominantly related to changes in energy metabolism and mitochondria functions in TgHD caused by the presence of transgenic human mutated huntingtin [15].

\subsection{Further markers of HD progression}

The Htt protein is part of many cell processes and it interacts with various proteins in cells [16]. Different markers can be used for characterization of HD progression in large animal models. Typical markers in the brain include medium-sized spiny neuron marker (DARPP32), marker for activated microglia (IBA1), an astrocyte marker (GFAP), marker associated with cell apoptosis (Cas3), markers of Golgi complex (ACBD3 and RHES) and marker of neuronal secretion (BDNF). We use coronal brain section containing striatum for immunohistochemical staining and measure intensity of the labelling in different brain areas: (1) motor cortex, (2) somatosensory cortex, (3) insular cortex, (4) caudate nucleus and (5) putamen, according to 3D view model of pig brain (Figure 4).

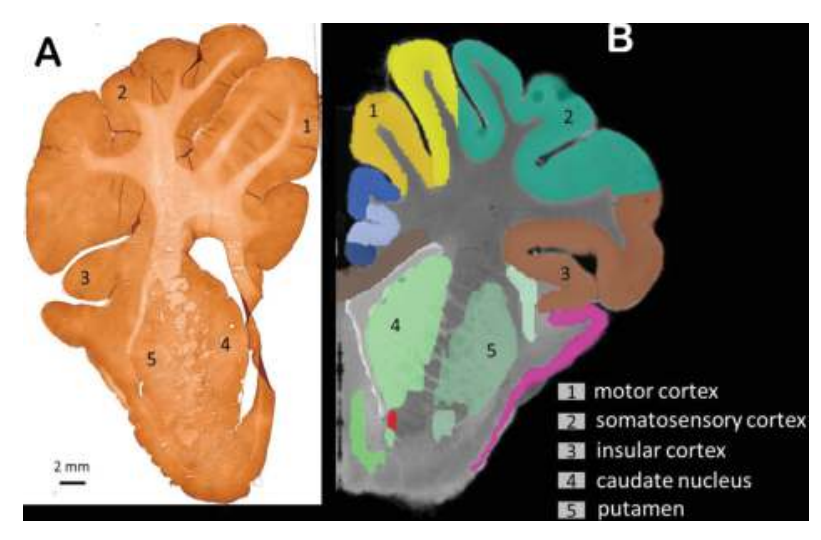

Figure 4. Identification of brain regions in porcine coronal brain section for evaluation. (A) Digitalized section staining with interest antibody. (B) Virtual section from 3D view model of pig brain (from program 3D slicer-slicer.org). 1. Motor cortex, 2. somatosensory cortex, 3. insular cortex, 4. caudate nucleus and 5. putamen.

Among the markers tested, medium-sized spiny neuron marker (DARPP32) was shown to be decreased in the striatum of our transgenic minipig model at 16 months [5]. DARPP32 mediates the response of medium-sized spiny neurons localized in the striatum to the activation of a dopamine receptor D1 [47]. The loss of DARPP32 was also shown in the brain of 7-month-old ovine transgenic HD model [2]. These results are in agreement with loss of D1 receptor detected in HD patients [48].

Next, apoptosis can be detected by higher expression of caspases. Caspase 3 was elevated in brain of HD minipig (N208-105Q), which died 53 h after birth [4]. Caspase 3 belongs to effectors 
of apoptosis, and its activation mediates apoptotic cell death in HD [49]. Activated caspase 3 was also found in post-mortem human HD brain [50].

\section{Immune response in central nervous system (CNS) and in the periphery}

The principal aim of current research in Huntington's disease is focused on detection of the pre-manifest disease stages [51].

Not all aberrant changes in HD are secondary to the neuronal dysfunction, but they might be caused by the expression of mutant $\mathrm{Htt}$ in the peripheral tissues [52, 53]. It is known that the immune system is implicated in the pathogenesis of HD [54-57]. Therefore, inflammation is a growing area of research in HD.

The ubiquitously expressed mHtt may likely cause parallel inflammation in central nervous system (CNS) and in the periphery [58, 59] (Figure 5). Björkqvist et al. showed that immunomodulatory molecules IL- 6 and IL- 8 are over-expressed in the striatum and also in plasma of HD patients [54].

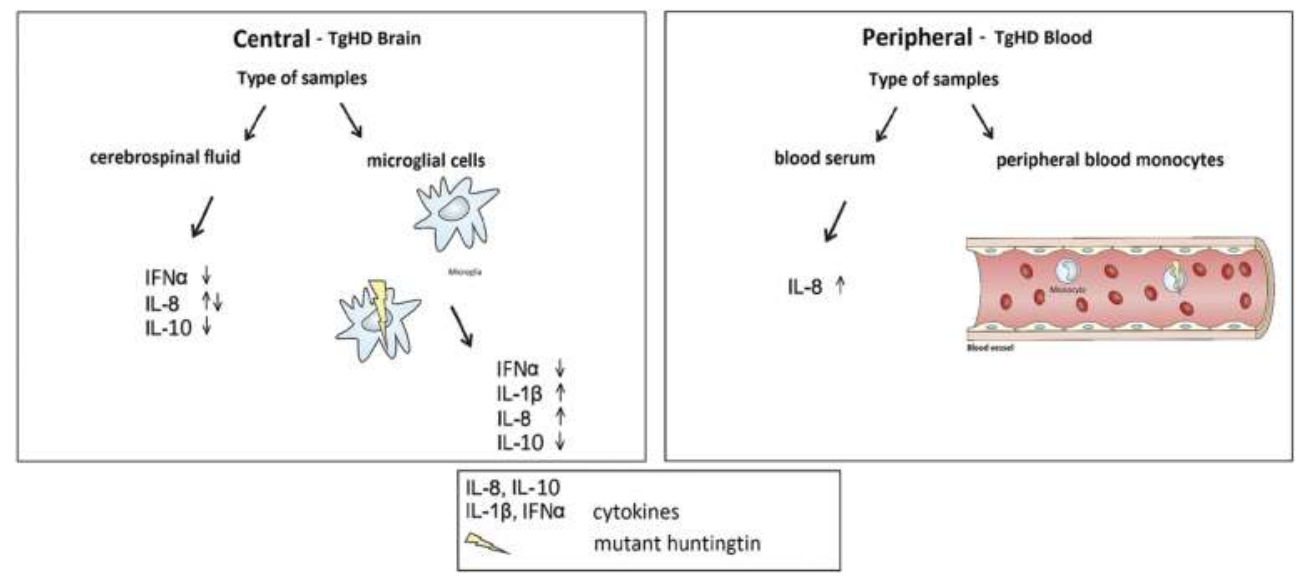

Figure 5. Immune activation in TgHD minipigs. The activation of immune system induced by mutant huntingtin, in CNS (central) and in the periphery (peripheral) as well.

In order to identify the mechanisms of immune system dysfunction in HD, our porcine model of HD was used [60]. The advantage of this large animal model is the ability to obtain samples from WT and transgenic TgHD animals with similar genetic background. The CNS immune response was measured by the levels of cytokines in CSF and in the secretome (in culture media) of cerebellar microglial cells. The inflammation in the periphery was simultaneously measured by the cytokine levels in serum and culture media of CD14+ blood monocytes. The samples were collected from WT and TgHD minipigs at the age of 9-36 months. TgHD minipigs at this age represent the pre-symptomatic stage of $\mathrm{HD}$, thus offering the opportunity 
to study early pathologic mechanisms before the disease onset. Multiplexing bead-based assay allowing the measurement of seven different porcine cytokines (IL-1 $\beta$, IL-4, IL-8, IL-10, IFN $\gamma$, IFN $\alpha$ and TNF $\alpha$ ) was used. Microglia secretomes and CSF of TgHD minipigs showed decreased levels of IFN $\alpha$ and IL-10, whereas microglia secretome as well indicated increased levels of IL-8 and IL-1 $\beta$ compared to WT animal controls. The difference in cytokine production in TgHD vs. WT samples is possibly caused by the presence of mutated Htt in TgHD microglia. Furthermore, increased levels of IL- 8 were observed in TgHD serum samples.

This study suggested IFN $\alpha$, IL-10, IL- 8 and IL-1 $\beta$ as promising biomarkers reflecting immunopathological mechanisms of HD minipig model in the disease pre-symptomatic stage. Identifications of these candidate biomarkers in CSF and serum could be valuable for monitoring the HD progression and therapy. Better understanding of the earliest changes in brain tissue as well as in periphery system may lead to preventive or disease-modifying therapies [60].

\section{Behavioural, motoric and cognitive studies}

Behaviour is an important parameter in several neuroscience disciplines. People with HD have great difficulties with coordination, focusing and learning. The symptoms include involuntary chorea-like movements, poor balance, slurred speech, difficulty swallowing, cognitive difficulty and personality change. HD patients often have depression, anxiety, irritability and apathy. Interestingly, not all symptoms are experienced by all patients [61]. Mice models also exhibit difficulties in a number of tasks, namely swimming, beam traversing and maintaining balance on the rota rod at the fastest rotating speeds [62].

Several behavioural, cognitive and motoric tests using mainly F3 generation of Libechov TgHD and WT minipigs were established in George Huntington's Institute in Muenster [61]. The list includes GAITRite automated acquisition system, a carpet denoting walking, which can be used to detect imbalance and disturbance in walking. Hurdle test aims also at assessing motor coordination of gait and tongue, and coordination test detects tongue protrusion. A colour discrimination test, dominance test and a startbox back and forth test assess cognitive deficits in minipigs. The tests are easily done with Libechov TgHD and WT minipigs, and they are reproducible. Nevertheless, this group of tested minipigs, where the oldest were three and half years old, did not show any differences in performing above-mentioned tests in TgHD compared to WT. We have also performed some of these tests in Libechov using the oldest F0 and F1 generations of the minipigs. We established several additional tests involving a little stressful situation, for example, climbing on a balance beam or a pullback test. The oldest two animals, starting at 5 years, exhibit motoric defects and anxiety behaviour (data not published); however, this does not constitute a significant group of animals yet.

Additionally, we established, together with Technical University in Prague, vocalizationgrunting test of the TgHD minipigs (Figure 6). Transgenic mHtt songbirds (145Q), created by lentiviral injection into the embryos, reflect severe vocal disorders associated with HD neuropathology [63]. It is also known that large majority of HD patients, more than $90 \%$, 
develop voice and speech dysfunction, abnormalities in speech timing, articulation deficits and irregular loudness [64]. Some of these symptoms start already before the onset of the disease [64-68]. Pigs have similar articular organs as humans, and thus, similar motor disturbances like rigidity, chorea and bradykinesia can be expected. A preliminary data and the experiment set-up were published [69].

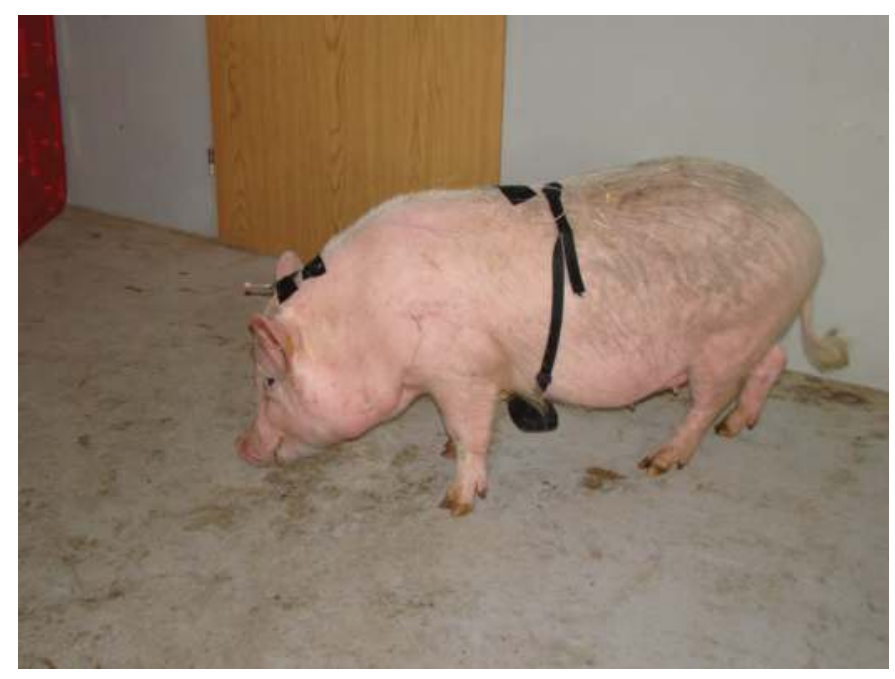

Figure 6. A pig with a fixed microphone. Microphone records sounds with the MP3 player. These recordings are then transferred to a computer for audio analysis.

We observed three types of grunting:

1. Single grunts - associated with investigatory behaviour or contact calls in group.

2. Single squeals - higher level of arousal, but function is similar as single grunts.

3. Rapidly repeated grunts - appear to have either a greeting or threat function [69].

Several minutes long recordings of different grunting were taped, and the results will be analysed using acoustic software Praat used in evaluation of humans [70].

\subsection{Telemetry}

The long-term collection of neurobehavioral and other physiological data using telemetry devices represents a critical component of differently focused animal studies. Such devices have to be implanted in a location that is safe, well tolerated and functional. The major advantage of telemetry approach is the collection of biopotentials from freely moving experimental animals without the presence of disturbing factors - e.g. researchers - during different time periods (even in nonstop mode) that results into relatively objective data, which can be analysed by software. 
Pigs, piglets and especially minipigs represent convenient large animal models for biomedical studies also in relation to the telemetry approach thanks to their relatively small size, characterized health status and ease of training and handling [71-74].

Important early features identified in HD patients include sleep deficits and disrupted circadian organization; these also correlate with symptom severity. Similar observations were also made in R6/2 mouse model [75] and ovine model of HD [76]. Mutated Htt action and loss of wild-type Htt function affect not only the brain structures, but also peripheral tissues or organ systems like testes, heart, pancreas, skeletal muscle, etc. [11, 52, 77, 78]. A major cause of death in HD patients includes heart disease. Moreover, in the R6/1 model, strong dysfunction of the autonomic cardiac nervous system was detected resulting in cardiac arrhythmias and sudden death [79].

The telemetry approach was applied to detect and analyse a pathological pattern in physical activity of TgHD boars at the age of 3 years [80]. In this study, we included five TgHD and five wild-type (WT) animals for comparison. The physical activity was measured by the telemetric system rodentPACK2 (emka TECHNOLOGIES, France), whereas transmitters were placed into the collar. For reducing collar influence on minipig activity, the boars wore collars without transmitters a few days before beginning of the study. The analysis showed significant decrease in total acceleration representing physical activity in TgHD boars between 4:40 and 5:30 a.m. (after night sleep and before morning feeding) in comparison with WT boars (Figure 7). This could be explained with disturbed energy metabolism.

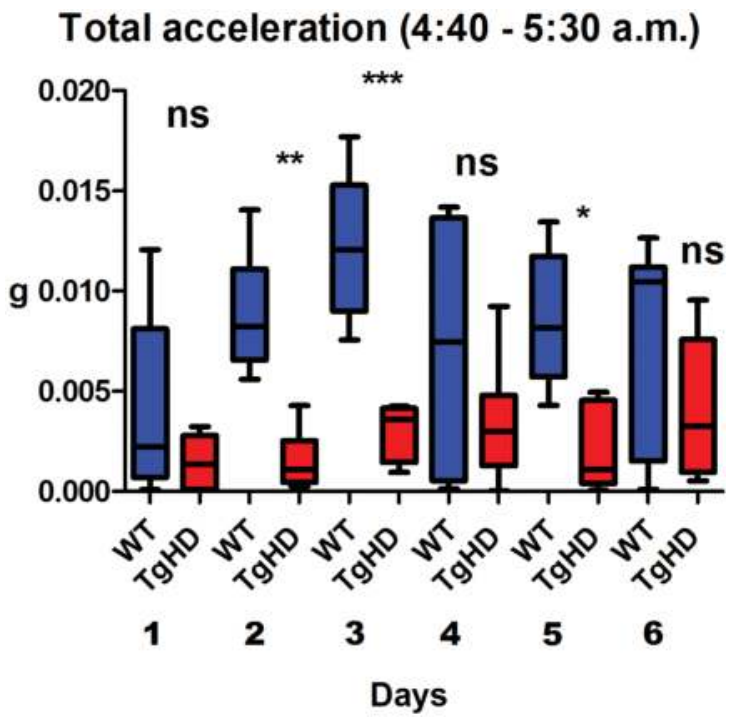

Figure 7. Total acceleration of TgHD and WT animals between 4:40 and 5:30 a.m. during six following days. Each column (blue-WT, red-TgHD) represents averaged total acceleration of five animals (TgHD or WT). Reprinted with permission of CzMA JEP adopted from CeskSlovNeurol N2015; 78/111 (Suppl 2). 


\section{Perspective and engineering of new models}

A variety of HD animal models has been already engineered; nevertheless, it is necessary to generate improved models. While each model has some of the typical markers of HD, no model recapitulates the full phenotype of HD patients. The advantage of HD for model engineering is the conditionality of a single gene by the mutation in the polyQ stretch of HTT gene. However, the mHtt cytotoxicity may include more factors influencing the disease development, and it is not yet clear what mechanism causes the HD pathology. So the question is what should the ideal HD model carry out in the genome? New approaches of gene editing allow the sequence-specific targeting of genome and the design of modifications in the endogenous HTT locus. Recently, the CRISPR/Cas9 system has become the most widespread system due to the universality of the available engineered nucleases, and there are a lot of recent improvements in its application.

The CRISPR/Cas9 system enables the site-specific modification at a desired region in the genome. This system, just as programmable nucleases, namely the transcription activator-like effector nuclease (TALEN) and the zinc-finger nuclease (ZFN), is feasible to combine with the several genetic engineering applications. The somatic cell nuclear transfer (SCNT) together with the microinjection into the zygote belongs between the widely employed methods of generating genetically modified porcine models. There are advantages and disadvantages in both approaches. The SCNT gives us an opportunity to select the genetically modified cells and transfer only embryos with modified genome into a recipient sow. On the other side, genetic diversity of offspring is conserved using microinjection into the zygotes [81]. First genome edited pig was generated in 2011 followed by many other porcine knockout (KO) models [82]. However, the generation of porcine knock-in (KI) model remains a huge challenge for the researchers due to certain limitations such as incompetence of porcine embryonic stem cells and induced pluripotent stem cells for SCNT [83], founder mosaicism [84], abnormal epigenetic programming resulting in prenatal and early postnatal death [85] and other technical difficulties. Nevertheless, Peng et al. [86] successfully generated CRISPR/Cas9mediated knock-in pig. They inserted human albumin (ALB) cDNA into porcine ALB locus. This approach is giving a hope to replace one wild-type allele and to create the DNA configuration of HD patients.

\section{Conclusion}

The Libechov TgHD minipig constitutes an animal model with slowly progressing phenotype similar to HD patients. The piglets are born with no evident defects, and the first detectable changes start at the age of 13 months. The TgHD boars have testicular degeneration and show a reproductive failure with low number of spermatozoa incompetent to penetrate the oocyte. MRS analysis of testes detected a significant reduction in relative PDE concentration in testicular parenchyma of 24-month-old TgHD boars, which could be related to changes in sperm motility. At 16 months, we detect lower expression of DARPP32 in TgHD striatum. 
Fragmentation of $\mathrm{mHtt}$, especially in the brain and testes, starts at 2 years and increases with age, similar to the appearance of oligomeric smears containing $\mathrm{mHtt}$. The study of immune response in CSF and periphery suggested IFN $\alpha$, IL-10, IL-8 and IL-1 $\beta$ as promising biomarkers reflecting immunopathological mechanisms of the pre-symptomatic stage of HD in the minipig model. Several non-invasive methods have been established in Libechov as well as in the George Huntington Institute, testing motor coordination, behaviour and cognition of TgHD minipigs. These methods can be applied to porcine models generated for various neurological diseases. MRI and MRS are the methods of choice to track any changes in clinical studies. Thus, relevant values obtained in porcine preclinical MRI studies can be easily translated to clinics. Moreover, we have already described some changes in the preclinical stage, namely significant decrease in total creatine $(\mathrm{tCr})$ in the brains of 2-year-old TgHD animals. Also, telemetric studies showed differences in physical activity patterns of 3-year-old TgHD compared to WT minipigs between 4:40 and 5:30 a.m. The oldest two animals, starting at the age of 5 years, show motoric defects and accentuated anxiety behaviour. Therefore, we expect the clinical onset of $\mathrm{HD}$ in TgHD animals with the $\mathrm{N}$-terminal part of human $\mathrm{mHtt}$ at the age of approximately 6 years. This fact needs to be confirmed using a higher number of animals reaching this age. Meanwhile, due to the availability of novel transgenic technologies, we are attempting to generate a knock-in humanized minipig in order to produce even better large animal model for HD.

The large animal HD models are the missing link between the mouse models and human patients; they may identify early dysfunctions of HD pathophysiology that could be used for future HD treatment approaches. There is an urgent need to identify specific biomarkers and to generate disease-modifying treatments that could be able to delay the HD onset or even reverse the disease progression [87, 88]. Our porcine HD model described here has already been used in preclinical testing of therapeutic strategies to reduce the amount of $\mathrm{mHtt}$ and thus proved to be important in this field.

\section{Acknowledgements}

This study was supported by National Sustainability Programme, project number LO1609 (Czech Ministry of Education, Youth and Sports).

\section{Author details}

Petra Rausova, Petra Vochozkova, Daniela Vidinska, Eva Hrnciarova, Bozena Bohuslavova, Monika Macakova, Ivona Valekova, Stefan Juhas, Taras Ardan, Petr Solc, Jan Motlik and Zdenka Ellederova*

*Address all correspondence to: ellederova@iapg.cas.cz

Institute of Animal Physiology and Genetics, ASCR, Libechov, Czech Republic 


\section{References}

[1] Yang S-H, Cheng P-H, Banta H, Piotrowska-Nitsche K, Yang J-J, Cheng EC, et al. Towards a transgenic model of Huntington's disease in a non-human primate. Nature 2008; 453(7197): 921-4. doi:10.1038/nature06975

[2] Jacobsen JC, Bawden CS, Rudiger SR, McLaughlan CJ, Reid SJ, Waldvogel HJ, et al. An ovine transgenic Huntington's disease model. Hum Mol Genet 2010; 19(10): 1873-82. doi:10.1093/hmg/ddq063

[3] Uchida M, Shimatsu Y, Onoe K, Matsuyama N, Niki R, Ikeda JE, et al. Production of transgenic miniature pigs by pronuclear microinjection. Transgenic Res 2001; 10(6): 577-82. doi:10.1023/A:1013059917280

[4] Yang D, Wang CE, Zhao B, Li W, Ouyang Z, Liu Z, et al. Expression of Huntington's disease protein results in apoptotic neurons in the brains of cloned transgenic pigs. Hum Mol Genet 2010; 19(20): 3983-94. doi:10.1093/hmg/ddq313

[5] Baxa M, Hruska-Plochan M, Juhas S, Vodicka P, Pavlok A, Juhasova J, et al. A transgenic minipig model of Huntington's disease. J Huntingtons Dis 2013; 2(1): 47-68. doi: 10.3233/JHD-130001

[6] Vodicka P, Smetana K, Dvorankova B, Emerick T, Xu YZ, Ourednik J, et al. The miniature pig as an animal model in biomedical research. Ann N Y Acad Sci 2005; 1049: 161-71. doi:10.1196/annals.1334.015

[7] Lunney JK. Advances in swine biomedical model genomics. Int J Biol Sci 2007; 3(3): 179-84. doi:10.7150/ijbs.3.179

[8] Swindle MM, Makin A, Herron AJ, Clubb FJ, Frazier KS. Swine as models in biomedical research and toxicology testing. Vet Pathol 2012; 49(2): 344-56. doi:10.1177/03009858 11402846

[9] Saudou F, Humbert S. The biology of Huntingtin. Neuron 2016; 89(5): 910-26. doi: 10.1016/j.neuron.2016.02.003

[10] Hannan AJ, Ransome MI. Deficits in spermatogenesis but not neurogenesis are alleviated by chronic testosterone therapy in R6/1 Huntington's disease mice. J Neuroendocrinol 2012; 24(2): 341-56. doi:10.1111/j.1365-2826.2011.02238.x

[11] Sathasivam K, Hobbs C, Turmaine M, Mangiarini L, Mahal A, Bertaux F, et al. Formation of polyglutamine inclusions in non-CNS tissue. Hum Mol Genet 1999; 8(5): 81322. doi:10.1093/hmg/8.5.813

[12] Rath D, Niemann H. In vitro fertilization of porcine oocytes with fresh and frozenthawed ejaculated or frozen-thawed epididymal semen obtained from identical boars. Theriogenology 1997; 47(4): 785-93. doi:10.1016/S0093-691X(97)00034-4 
[13] O'Donnell L, Nicholls PK, O'Bryan MK, McLachlan RI, Stanton PG. Spermiation: the process of sperm release. Spermatogenesis 2011; 1(1): 14-35. doi:10.4161/spmg.1.1. 14525

[14] Macakova M, Bohuslavova B, Vochozkova P, Pavlok A, Sedlackova M, Vidinska D, et al. Mutated huntingtin causes testicular pathology in transgenic minipig boars. Neurodegener Dis 2016; 16(3-4): 245-59. doi:10.1159/000443665

[15] Jozefovicova M, Herynek V, Jiru F, Dezortova M, Juhasova J, Juhas S, et al. Minipig model of Huntington's disease: (1)H magnetic resonance spectroscopy of the brain. Physiol Res 2016; 65(1): 155-63.

[16] Zuccato C, Valenza M, Cattaneo E. Molecular mechanisms and potential therapeutical targets in Huntington's disease. Physiol Rev 2010; 90(3): 905-81. doi:10.1152/physrev. 00041.2009

[17] Ogen-Shtern N, Ben David T, Lederkremer GZ. Protein aggregation and ER stress. Brain Res 2016; 1648(Pt B): 658-66. doi:10.1016/j.brainres.2016.03.044.

[18] Arrasate M, Mitra S, Schweitzer ES, Segal MR, Finkbeiner S. Inclusion body formation reduces levels of mutant huntingtin and the risk of neuronal death. Nature 2004; 431(7010): 805-10. doi:10.1038/nature02998

[19] Lajoie P, Snapp EL. Formation and toxicity of soluble polyglutamine oligomers in living cells. PLoS One 2010; 5(12). doi:10.1371/journal.pone.0015245

[20] Wong BK, Ehrnhoefer DE, Graham RK, Martin DD, Ladha S, Uribe V, et al. Partial rescue of some features of Huntington disease in the genetic absence of caspase- 6 in YAC128 mice. Neurobiol Dis 2015; 76: 24-36. doi:10.1016/j.nbd.2014.12.030

[21] Hoffner G, Souès S, Djian P. Aggregation of expanded huntingtin in the brains of patients with Huntington disease. Prion 2007; 1(1): 26-31. doi:10.4161/pri.1.1.4056

[22] Chambers W. Histo-chemical Study of the Senile Plaques of Redlich-Fischer [Etude histo-chimique des plaques seniles.](Journ. de Neur. et Psychiat., November, 1927.) Divry, P. Br J Psychiatry 1928; 74(306): 533. doi:10.1192/bjp.74.306.533-a.

[23] Frid P, Anisimov SV, Popovic N. Congo red and protein aggregation in neurodegenerative diseases. Brain Res Rev 2007; 53(1): 135-60. doi:10.1016/j.brainresrev.2006.08.001

[24] Wanker EE, Scherzinger E, Heiser V, Sittler A, Eickhoff H, Lehrach H. Membrane filter assay for detection of amyloid-like polyglutamine-containing protein aggregates. Methods Enzymol 1999; 309(1983): 375-86. doi:10.1016/S0076-6879(99)09026-6

[25] Sathasivam K, Lane A, Legleiter J, Warley A, Woodman B, Finkbeiner S, et al. Identical oligomeric and fibrillar structures captured from the brains of R6/2 and knock-in mouse models of Huntington's disease. Hum Mol Genet 2009; 19(1): 65-78. doi:10.1093/hmg/ $\operatorname{ddp} 467$ 
[26] Laferrière F, Tixador P, Moudjou M, Chapuis J, Sibille P, Herzog L, et al. Quaternary structure of pathological prion protein as a determining factor of strain-specific prion replication dynamics. PLoS Pathog 2013; 9(10). doi:10.1371/journal.ppat.1003702

[27] Weiss A, Klein C, Woodman B, Sathasivam K, Bibel M, Régulier E, et al. Sensitive biochemical aggregate detection reveals aggregation onset before symptom development in cellular and murine models of Huntington's disease. J Neurochem 2008; 104(3): 846-58. doi:10.1111/j.1471-4159.2007.05032.x

[28] Vidinská D, Motlík J, Ellederová Z. Different forms of Huntingtin in the most affected organs; brain and testes of transgenic minipigs. Česká a Slov Neurol a Neurochir 2015; 78/111(S2): 66-9. doi:10.14735/amcsnn20152S66

[29] Bechtel N, Scahill RI, Rosas HD, Acharya T, Van den Bogaard SJ, Jauffret C, et al. Tapping linked to function and structure in premanifest and symptomatic Huntington disease. Neurology 2010; 75(24): 2150-60. doi:10.1212/WNL.0b013e3182020123

[30] Delmaire C, Dumas EM, Sharman MA, van den Bogaard SJ, Valabregue R, Jauffret C, et al. The structural correlates of functional deficits in early huntington's disease. Hum Brain Mapp 2013; 34(9): 2141-53. doi:10.1002/hbm.22055

[31] Scahill RI, Hobbs NZ, Say MJ, Bechtel N, Henley SM, Hyare H, et al. Clinical impairment in premanifest and early Huntington's disease is associated with regionally specific atrophy. Hum Brain Mapp 2013; 34(3): 519-29. doi:10.1002/hbm.21449

[32] Hölzner E, Baxa M, Nagelmann N, Marcegaglia M, Weber N, Göpfert N, et al. TRACK$\operatorname{tgHD}$ minipig - introduction of a longitudinal tgHD minipig phenotyping study using MRI, motor and cognitive endpoints. Basal Ganglia 2013; 3(1). doi:10.1016/j.baga. 2013.01.016

[33] Sundgren PC, Jennings J, Attwood JT, Nan B, Gebarski S, McCune WJ, et al. MRI and 2D-CSI MR spectroscopy of the brain in the evaluation of patients with acute onset of neuropsychiatric systemic lupus erythematosus. Neuroradiology 2005; 47(8): 576-85. doi:10.1007/s00234-005-1371-y

[34] Van den Bogaard SJ, Dumas EM, Teeuwisse WM, Kan HE, Webb A, Roos RA, et al. Exploratory 7-Tesla magnetic resonance spectroscopy in Huntington's disease provides in vivo evidence for impaired energy metabolism. J Neurol 2011; 258(12): 2230-9. doi: 10.1007/s00415-011-6099-5

[35] Sturrock A, Laule C, Decolongon J, Dar Santos R, Coleman AJ, Creighton S, et al. Magnetic resonance spectroscopy biomarkers in premanifest and early Huntington disease. Neurology 2010; 75(19): 1702-10. doi:10.1212/WNL.0b013e3181fc27e4

[36] van den Bogaard SJ, Dumas E, van der Grond J, van Buchem M, Roos R. MRI biomarkers in Huntington's disease. Front Biosci (Elite Ed) 2012; 4: 1910-25. doi:10.1093/carcin/ bgs015. 
[37] Unschuld PG, Edden RA, Carass A, Liu X, Shanahan M, Wang X, et al. Brain metabolite alterations and cognitive dysfunction in early Huntington's disease. Mov Disord 2012; 27(7): 895-902. doi:10.1002/mds.25010

[38] Tkac I, Henry PG, Zacharoff L, Wedel M, Gong W, Deelchand DK, et al. Homeostatic adaptations in brain energy metabolism in mouse models of Huntington disease. J Cereb Blood Flow Metab 2012; 32(11): 1977-88. doi:10.1038/jcbfm.2012.104

[39] Zacharoff L, Tkac I, Song Q, Tang C, Bolan PJ, Mangia S, et al. Cortical metabolites as biomarkers in the R6/2 model of Huntington's disease. J Cereb Blood Flow Metab 2012; 32(3): 502-14. doi:10.1038/jcbfm.2011.157

[40] Reynolds NC, Prost RW, Mark LP. Heterogeneity in 1H-MRS profiles of presymptomatic and early manifest Huntington's disease. Brain Res 2005; 1031(1): 82-9. doi: 10.1016/j.brainres.2004.10.030

[41] Rotondo E, Bruschetta G, Saccà A, Bramanti P, Di Pasquale MR. Straightforward relative quantitation and age-related human standards of $\mathrm{N}$-acetylaspartate at the centrum semiovale level by CSI 1H-MRS. Magn Reson Imaging 2003; 21(9): 1055-60. doi:10.1016/ S0730-725X(03)00211-X

[42] van Oostrom JC, Sijens PE, Roos RA, Leenders KL. 1H magnetic resonance spectroscopy in preclinical Huntington disease. Brain Res 2007; 1168(1): 67-71. doi:10.1016/ j.brainres.2007.05.082

[43] Gomez-Anson B, Alegret M, Munoz E, Sainz A, Monte GC, Tolosa E. Decreased frontal choline and neuropsychological performance in preclinical Huntington disease. Neurology 2007; 68(12): 906-10. doi:10.1212/01.wnl.0000257090.01107.2f

[44] van den Bogaard SJ, Dumas EM, Teeuwisse WM, Kan HE, Webb A, van Buchem MA, et al. Longitudinal metabolite changes in Huntington's disease during disease onset. J Huntingtons Dis 2014; 3(4): 377-86. doi:10.3233/JHD-140117

[45] Odish OF, Leemans A, Reijntjes RH, van den Bogaard SJ, Dumas EM, Wolterbeek R, et al. Microstructural brain abnormalities in Huntington's disease: a two-year follow-up. Hum Brain Mapp 2015; 36(6): 2061-74. doi:10.1002/hbm.22756

[46] Odish OF, Caeyenberghs K, Hosseini H, van den Bogaard SJ, Roos RA, Leemans A. Dynamics of the connectome in Huntington's disease: a longitudinal diffusion MRI study. NeuroImage Clin 2015; 9: 32-43. doi:10.1016/j.nicl.2015.07.003

[47] Ouimet CC, Greengard P. Distribution of DARPP-32 in the basal ganglia: an electron microscopic study. J Neurocytol 1990; 19(1): 39-52. doi:10.1007/BF01188438

[48] Li SH, Li XJ. Huntingtin-protein interactions and the pathogenesis of Huntington's disease. Trends Genet 2004; 20(3): 146-54. doi:10.1016/j.tig.2004.01.008

[49] Friedlander RM. Apoptosis and caspases in neurodegenerative diseases. N Engl J Med 2003; 348(14): 1365-75. doi:10.1056/NEJMra022366 
[50] Hermel E, Gafni J, Propp SS, Leavitt BR, Wellington CL, Young JE, et al. Specific caspase interactions and amplification are involved in selective neuronal vulnerability in Huntington's disease. Cell Death Differ 2004; 11(4): 424-38. doi:10.1038/sj.cdd.4401358

[51] Henley SM, Bates GP, Tabrizi SJ. Biomarkers for neurodegenerative diseases. Curr Opin Neurol 2005; 18: 698-705. doi:10.1097/01.wco.0000186842.51129.cb

[52] van der Burg JM, Björkqvist M, Brundin P. Beyond the brain: widespread pathology in Huntington's disease. Lancet Neurol 2009; 8(8): 765-74. doi:10.1016/S1474-4422(09) 70178-4

[53] Sassone J, Colciago C, Cislaghi G, Silani V, Ciammola A. Huntington's disease: the current state of research with peripheral tissues. Exp Neurol 2009; 219(2): 385-97. doi: 10.1016/j.expneurol.2009.05.012

[54] Björkqvist M, Wild EJ, Thiele J, Silvestroni A, Andre R, Lahiri N, et al. A novel pathogenic pathway of immune activation detectable before clinical onset in Huntington's disease. J Exp Med 2008; 205(8): 1869-77. doi:10.1084/jem.20080178

[55] Dalrymple A, Wild EJ, Joubert R, Sathasivam K, Bjorkqvist M, Petersen A, et al. Proteomic profiling of plasma in Huntington's disease reveals neuroinflammatory activation and biomarker candidates. J Proteome Res 2007; 6(7): 2833-40. doi:10.1021/ pr0700753

[56] Frank-Cannon TC, Alto LT, McAlpine FE, Tansey MG. Does neuroinflammation fan the flame in neurodegenerative diseases? Mol Neurodegener 2009; 4: 47. doi:10.1186/17501326-4-47

[57] Kotrcova E, Jarkovska K, Valekova I, Zizkova M, Motlik J, Gadher SJ, et al. Challenges of Huntington's disease and quest for therapeutic biomarkers. Proteomics-Clin Appl 2015; 9(1-2): 147-58. doi:10.1002/prca.201400073

[58] Möller T. Neuroinflammation in Huntington's disease. J Neural Transm 2010; 117(8): 1001-8. doi:10.1007/s00702-010-0430-7

[59] Ellrichmann G, Reick C, Saft C, Linker RA. The role of the immune system in Huntington's disease. Clin Dev Immunol 2013; 2013: 541259. doi:10.1155/2013/541259

[60] Valekova I, Jarkovska K, Kotrcova E, Bucci J, Ellederova Z, Juhas S, et al. Revelation of the IFN $\alpha$, IL-10, IL-8 and IL-1 $\beta$ as promising biomarkers reflecting immuno-pathological mechanisms in porcine Huntington's disease model. J Neuroimmunol 2016; 293: 71-81. doi:10.1016/j.jneuroim.2016.02.012

[61] Schramke S, Schuldenzucker V, Schubert R, Frank F, Wirsig M, Ott S, et al. Behavioral phenotyping of minipigs transgenic for the Huntington gene. J Neurosci Methods 2015. doi:10.1016/j.jneumeth.2015.11.013

[62] Luesse HG, Schiefer J, Spruenken A, Puls C, Block F, Kosinski CM. Evaluation of R6/2 $\mathrm{HD}$ transgenic mice for therapeutic studies in Huntington's disease: behavioral testing 
and impact of diabetes mellitus. Behav Brain Res 2001; 126(1-2): 185-95. doi:10.1016/ S0166-4328(01)00261-3

[63] Liu W, Kohn J, Szwed SK, Pariser E, Sepe S, Haripal B, et al. Human mutant huntingtin disrupts vocal learning in transgenic songbirds. Nat Neurosci 2015; 18(11): 1617-22. doi:10.1038/nn.4133

[64] Rusz J, Klempr J, Tykalova T, Baborova E, Cmejla R, Ruzicka E, et al. Characteristics and occurrence of speech impairment in Huntington's disease: possible influence of antipsychotic medication. J Neural Transm 2014; 121(12): 1529-39. doi:10.1007/s00702014-1229-8

[65] Vogel AP, Shirbin C, Churchyard AJ, Stout JC. Speech acoustic markers of early stage and prodromal Huntington's disease: a marker of disease onset? Neuropsychologia 2012; 50(14): 3273-8. doi:10.1016/j.neuropsychologia.2012.09.011

[66] Rusz J, Klempir J, Baborova E, Tykalova T, Majerova V, Cmejla R, et al. Objective acoustic quantification of phonatory dysfunction in Huntington's disease. PLoS One 2013; 8(6). doi:10.1371/journal.pone.0065881

[67] Rusz J, Saft C, Schlegel U, Hoffman R, Skodda S. Phonatory dysfunction as a preclinical symptom of huntington disease. PLoS One 2014; 9(11). doi:10.1371/journal.pone. 0113412

[68] Skodda S, Schlegel U, Hoffmann R, Saft C. Impaired motor speech performance in Huntington's disease. J Neural Transm 2014; 121(4): 399-407. doi:10.1007/s00702-013$1115-9$

[69] Tykalova T, Hlavnicka J, Macakova M, Baxa M, Cmejla R, Motlik J, et al. Grunting in a genetically modified minipig animal model for Huntington's disease-pilot experiments. Česká a Slov Neurol a Neurochir 2015; 78/111(S2): 61-5. doi:10.14735/ amcsnn20152S61

[70] Boersma P. Praat, a system for doing phonetics by computer. Glot Int 59/10 2002; 3415. doi:10.1097/AUD.0b013e31821473f7

[71] Willens S, Cox DM, Braue EH, Myers TM, Wegner MD. Novel technique for retroperitoneal implantation of telemetry transmitters for physiologic monitoring in Göttingen minipigs (Sus scrofa domesticus). Comp Med 2014; 64(6): 464-70.

[72] Huppertz ND, Kirschner-Hermanns R, Tolba RH, Grosse JO. Telemetric monitoring of bladder function in female Göttingen minipigs. BJU Int 2015; 116(5): 823-32. doi: 10.1111/bju.13089

[73] Paskaranandavadivel N, Wang R, Sathar S, O'Grady G, Cheng LK, Farajidavar A. Multichannel wireless mapping of gastrointestinal serosal slow wave propagation. Neurogastroenterol Motil 2015; 27(4): 580-5. doi:10.1111/nmo.12515

[74] Krause A, Zebunke M, Bellmann O, Mohr E, Langbein J, Puppe B. Surgical implantation and functional assessment of an invasive telemetric system to measure autonomic responses in domestic pigs. Vet J 2016; 207: 140-6. doi:10.1016/j.tvj1.2015.10.050 
[75] Fisher SP, Black SW, Schwartz MD, Wilk AJ, Chen TM, Lincoln WU, et al. Longitudinal analysis of the electroencephalogram and sleep phenotype in the R6/2 mouse model of Huntington's disease. Brain 2013; 136(7): 2159-72. doi:10.1093/brain/awt132

[76] Morton JA, Rudiger SR, Wood NI, Sawiak SJ, Brown GC, Mclaughlan CJ, et al. Early and progressive circadian abnormalities in Huntington's disease sheep are unmasked by social environment. Hum Mol Genet 2014; 23(13): 3375-83. doi:10.1093/hmg/ddu047

[77] Dragatsis I, Levine MS, Zeitlin S. Inactivation of Hdh in the brain and testis results in progressive neurodegeneration and sterility in mice. Nat Genet 2000; 26(3): 300-6. doi: $10.1038 / 81593$

[78] Bano D, Zanetti F, Mende Y, Nicotera P. Neurodegenerative processes in Huntington's disease. Cell Death Dis 2011; 2(11): e228. doi:10.1038/cddis.2011.112

[79] Kiriazis H, Jennings NL, Davern P, Lambert G, Su Y, Pang T, et al. Neurocardiac dysregulation and neurogenic arrhythmias in a transgenic mouse model of Huntington's disease. J Physiol 2012; 590(Pt 22): 5845-60. doi:10.1113/jphysiol.2012.238113

[80] Pokorny M, Juhas S, Juhasova J, Klima J, Motlik J, Klempir J, et al. Telemetry physical activity monitoring in minipig's model of Huntington's disease. Česká a Slov Neurol a Neurochir 2015; 78/111(S2): 39-42. doi:10.14735/amcsnn20152S39

[81] Tan W, Proudfoot C, Lillico SG, Whitelaw CB. Gene targeting, genome editing: from Dolly to editors. Transgenic Res 2016; 1-15. doi:10.1007/s11248-016-9932-x

[82] Yang D, Yang H, Li W, Zhao B, Ouyang Z, Liu Z, et al. Generation of PPAR $\gamma$ monoallelic knockout pigs via zinc-finger nucleases and nuclear transfer cloning. Cell Res 2011; 21(6): 979-82. doi:10.1038/cr.2011.70

[83] Fan N, Chen J, Shang Z, Dou H, Ji G, Zou Q, et al. Piglets cloned from induced pluripotent stem cells. Cell Res 2013; 23(1): 162-6. doi:10.1038/cr.2012.176

[84] Lillico SG, Proudfoot C, Carlson DF, Stverakova D, Neil C, Blain C, et al. Live pigs produced from genome edited zygotes. Sci Rep 2013; 3: 2847. doi:10.1038/srep02847

[85] Long CR, Westhusin ME, Golding MC. Reshaping the transcriptional frontier: epigenetics and somatic cell nuclear transfer. Mol Reprod Dev 2014; 81(2): 183-93. doi: 10.1002/mrd.22271

[86] Peng J, Wang Y, Jiang J, Zhou X, Song L, Wang L, et al. Production of human albumin in pigs through CRISPR/Cas9-mediated knockin of human cDNA into swine albumin locus in the zygotes. Sci Rep 2015; 5: 16705. doi:10.1038/srep16705

[87] Andre R, Scahill RI, Haider S, Tabrizi SJ. Biomarker development for Huntington's disease. Drug Discov Today 2014; 19(7): 972-9. doi:10.1016/j.drudis.2014.03.002

[88] Hersch SM, Rosas HD. Biomarkers to enable the development of neuroprotective therapies for Huntington's disease Biomarkers to Enable the Development of Neuroprotective. Neurobiol Huntington's Dis Appl to Drug Discov 2011; 1-13. doi:10.1201/ EBK0849390005-c11 